\title{
Searching for evidence of diquark states using lattice QCD simulations
}

\author{
Ryutaro Fukuda* \\ RIKEN BNL Research Center, Brookhaven National Laboratory, Upton, NY 11973, USA \\ Department of Physics, The University of Tokyo, Tokyo 113-0033, Japan \\ E-mail: ir-fukudaent.phys.s.u-tokyo.ac.ip
}

\section{Philippe de Forcrand}

Institute for Theoretical Physics, ETH Zurich, CH-8093 Zurich, Switzerland

Theory Division, CERN, CH-1211 Geneva 23, Switzerland

\begin{abstract}
In recent years, exotic hadrons called $\mathrm{X}, \mathrm{Y}, \mathrm{Z}$ which cannot be explained by the quark model have been found one after another. The tetra-quark state, which is one of the typical scenarios to interpret the exotic state, is based on the existence of a diquark state. The discovery of a pentaquark state at CERN in 2015 also makes a diquark state even more important. Therefore, it is considered that a diquark state plays an important role in many kinds of QCD physics. We discuss existence of such diquarks from the viewpoint of a first principle calculation.
\end{abstract}

34th annual International Symposium on Lattice Field Theory

24-30 July 2016

University of Southampton, UK

${ }^{*}$ Speaker. 


\section{Introduction}

In this paper, evidence of diquark state [U] in view of a lattice QCD simulation is discussed. The most naive question for all people is why a diquark is important. There may be many answers to this question. For example, diquark picture is a hopeful candidate to explain exotic hadrons such as tetra-quark and penta-quark which cannot be explained naturally by quark model. In 2015, penta-quark was observed by LHC experiment at CERN [0]. This discovery makes diquark states even more important. Diquarks are also considered as the central ingredient of cold, dense matter where they condense to form a color superconductor. Analyses of finite density QCD have been developed not only phenomenologically but also quantitatively and we guess that new physically interesting things exist in this field. Taking this situation into consideration, we study if diquark states are for real using lattice QCD simulation.

Table 1: Classification of diquarks

\begin{tabular}{|c|c|c|c|}
\hline$J^{P}$ & Color & Flavor & Operator \\
\hline \hline $0^{+}$ & $\overline{3}$ & $\overline{3}$ & $q^{T} C \gamma_{5} q, q^{T} C \gamma_{4} \gamma_{5} q$ \\
\hline $1^{+}$ & $\overline{3}$ & 6 & $q^{T} C \vec{\gamma} q, q^{T} C \sigma_{i 4} q$ \\
\hline $0^{-}$ & $\overline{3}$ & 6 & $q^{T} C q, q^{T} C \gamma_{4} q$ \\
\hline $1^{-}$ & $\overline{3}$ & $\overline{3}$ & $q^{T} C \vec{\gamma} \gamma_{5} q, q^{T} C \sigma_{i j} q$ \\
\hline
\end{tabular}

Jaffe [B] proposed to divide diquarks into four classes as shown in Tablell, and to predict their properties using perturbation theory. Quantum properties of the diquarks are determined by combination of gamma matrices in diquark operator. In TableW, $q$ and $C$ are the quark field and the charge conjugation operator, respectively. The most important point for this classification is that the spin color effective interaction by one gluon exchange predicts a most stable diquark state called a good diquark. This good diquark corresponds to the color anti-triplet, flavor anti-symmetric and spin singlet positive parity channel. This phenomenological analysis implies that the mass of the good diquark is the lightest of all diquark states and the diquark correlation is enhanced in this good diquark channel. Therefore, we should check if this perturbative statement remains correct using lattice QCD simulation.

For this objective, we calculate diquark mass diferences between the good diquark and other diquarks and also analyze the density distribution of diquarks in 3-dimensional space through calculations of the density-density correlators.

\section{Frame work}

\subsection{Diquark states on a lattice}

Because a diquark state is a colored object, we need to consider how to deal with this state on a lattice. We have two possible choices. One is to introduce a gauge fixing method on the lattice. The other is to use some gauge invariant formulation. In our work, we adopt a gauge invariant strategy to respect the gauge invariant formalism of the lattice. To be more specific, we combine a diquark and a static quark into a color singlet. Namely, we consider a static-light-light baryon. 
This corresponds to look at a diquark in the background field of a static quark. Therefore, the relevant limit is to keep the static quark far from the light quarks. In this approach, a static-lightlight baryon operator $O_{\Gamma}(x)$ is given in the following expression with $\Gamma=1, \gamma_{\mu}, \gamma_{5}, \gamma_{5} \gamma_{\mu}, \sigma_{\mu \nu}$ for two light flavor quarks $u$ and $d$.

$$
O_{\Gamma}(x)=\varepsilon^{a b c}\left[u_{a}^{T}(x) C \Gamma d_{b}(x) \pm d_{a}^{T}(x) \Gamma u_{b}(x)\right] s_{c}(x),
$$

where the $+(-)$ sign corresponds to the flavor symmetric (anti-symmetric) combination and $s_{c}$ denotes the static quark.

\subsection{Diquark mass difference}

Using a static-light-light baryon, we can easily calculate the mass difference between diquarks. What we should do is to calculate the static-light-light baryon correlator $C_{\Gamma}(t)$ defined as follows in standard manner for usual hadron spectroscopy.

$$
C_{\Gamma}(t)=\sum_{\vec{x}}\left\langle O_{\Gamma}(\vec{x}, t) O_{\Gamma}^{\dagger}(\overrightarrow{0}, 0)\right\rangle
$$

In addition to a light quark propagator, a static quark propagator $D_{Q}^{-1}(x \mid y)$ from $y$ to $x$ is also needed to calculate this static-light-light baryon correlator. The static quark propagator can be analytically calculated in the following way for $x_{4}>y_{4}$ with the static quark mass $m_{Q}$.

$$
D_{Q}^{-1}(x \mid y)=\delta(\vec{x}-\vec{y})\left(\frac{1+\gamma_{4}}{2}\right)\left[\prod_{k=y_{4}}^{x_{4}-1} U_{4}(\vec{x}, k)\right]^{\dagger} e^{-m_{Q}\left(x_{4}-y_{4}\right)}
$$

The static-light-light baryon correlator decreases exponentially and this behavior is managed by the sum of the diquark mass and the static quark mass at large $t$ as follows.

$$
C_{\Gamma}(t) \propto e^{-[M(\text { diquark })+M(\text { static quark })] t} \quad(\text { at large } t)
$$

Consequently, we can extract the sum of the diquark mass and the static quark mass from this correlator for all diquark states. Then, we can calculate mass differences between two diquark states. While $m_{Q}$ itself is UV-divergent, and not physically meaningful, the mass differences between static-static-baryons in different channels are UV-finite. In an actual numerical simulation, the factor $e^{-m_{Q}\left(x_{4}-y_{4}\right)}$ can be dropped because this contribution is canceled in the calculation of diquark mass differences.

\subsection{Density-density correlator}

We consider the density-density correlator $C_{\Gamma}^{d d}\left(\overrightarrow{x_{1}}, \overrightarrow{x_{2}}, t\right)$ defined as follows with a density operator $\rho(x)$ [四].

$$
\begin{gathered}
C_{\Gamma}^{d d}\left(\vec{r}_{1}, \vec{r}_{2}, t\right)=\left\langle O_{\Gamma}(\overrightarrow{0}, 2 t) \rho\left(\vec{r}_{1}, t\right) \rho\left(\vec{r}_{2}, t\right) O_{\Gamma}^{\dagger}(\overrightarrow{0}, 0)\right\rangle \\
\rho(\vec{r}, t)=\bar{q}(\vec{r}, t) \gamma_{4} \rho(\vec{r}, t)
\end{gathered}
$$

We put a creation operator $O_{\Gamma}^{\dagger}$ for a static-light-light baryon at $t=0$, and an annihilation operator $O_{\Gamma}$ at $2 t$. In the middle of this time separation, we put two density operators and analyze the space 
distribution. In this calculation, we fix the distance $r$ from the static quark $Q$ to the two light quarks $r=\left|\vec{r}_{1}\right|=\left|\vec{r}_{2}\right|$. Therefore, this calculation method provides us with density-density correlators as a function of $\theta$ which is the angle between the two light quarks as shown in Fig.W. If the densitydensity correlator is enhanced when two light quarks approach each other, this can be interpreted as diquark attraction. On the other hand, if density-density correlator has no dependence on $\theta$, two light quarks have no specific interaction.

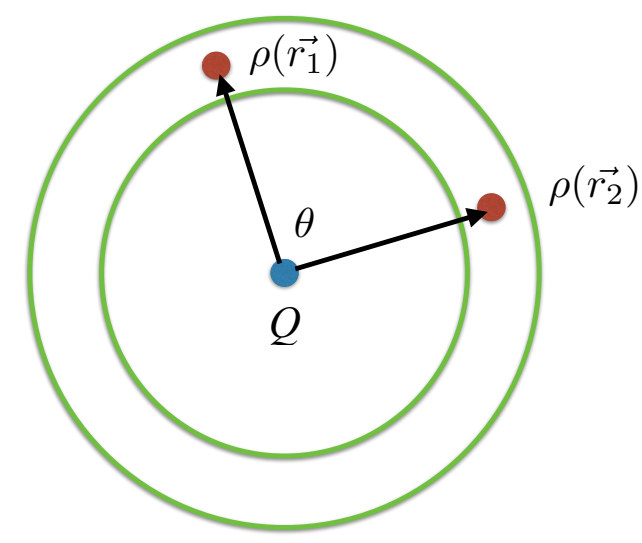

Figure 1: Density-density correlator

\section{Numerical results}

\subsection{Lattice design}

We use $O(a)$-improved 2-flavor Wilson fermions and standard Wilson gauge action. Thanks to CLS ensemble, we adopt a $32^{3} \times 64$ lattice with the ensemble A4 in Ref.[B]] and the pion mass is $380 \mathrm{MeV}$ in this setup.

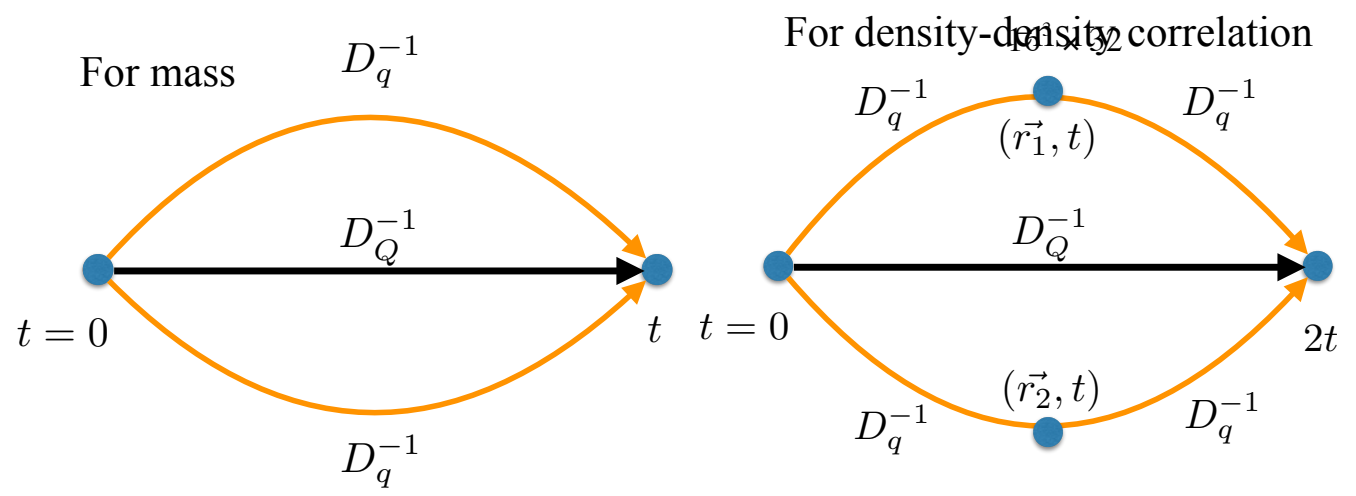

Figure 2: Calculation of effective mass difference and density-density correlator

For the calculation of diquark mass differences and density-density correlators, light quark propagators $D_{q}^{-1}$ and static quark propagators $D_{Q}^{-1}$ are needed as shown in Fig.】. For light quark 
propagators, source and sink Jacobi smearing with HYP smeared gauge links are used. Static quark propagators are also calculated with HYP smeared gauge links.

\subsection{Diquark mass difference}

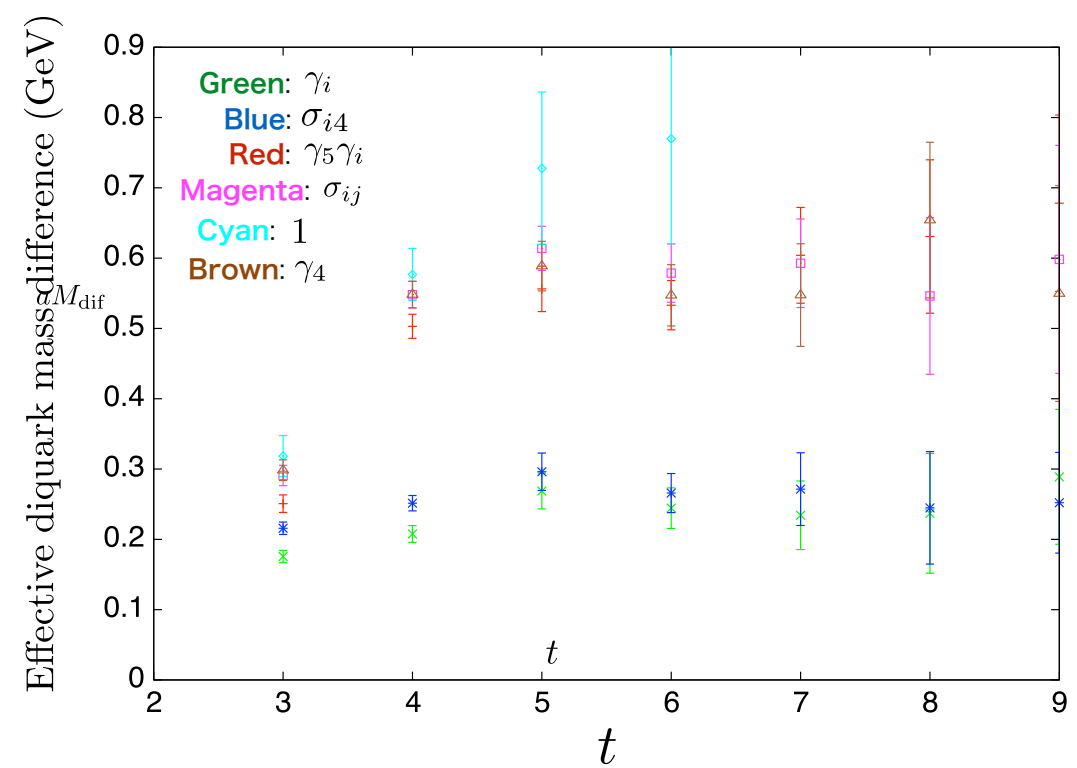

Figure 3: Effective mass difference between the good diquark and other diquarks

Figure 3 shows the effective mass differences between the good diquark and other diquarks. From this figure, we can say that the good diquark, the positive parity scalar, is the lightest of all diquark states as expected. The positive parity vector diquarks are degenerate and have clearly higher energy than the good diquark. Moreover, the negative parity scalars and vectors have much larger energy than the positive parity states. These statements are consistent with the prediction by the phenomenological analysis based on one gluon exchange.

\subsection{Space distribution of density-density correlator}

Figure 9 shows the space distribution of the density-density correlator for all diquark channels. Because a cubic lattice generally breaks rotational symmetry and distorts the uniform spherical distribution, we normalize the space distributions of the density-density correlator by the uniform lattice distribution to effectively remove this lattice artifact. Note that the horizontal axis is the value of $\cos \theta$, where $\theta$ denotes the angle between two light quarks (see Fig.W). We can clearly observe that the good diquark shows enhanced correlation when the two light quarks are close to each other. In contrast to the good diquark, no clear signal of interaction is observed in other diquark channels.

\section{Conclusion}

Using lattice QCD simulations, we can clearly observe that a good diquark is the lightest among all possible diquark states. In addition, we can also state that attraction between two light 


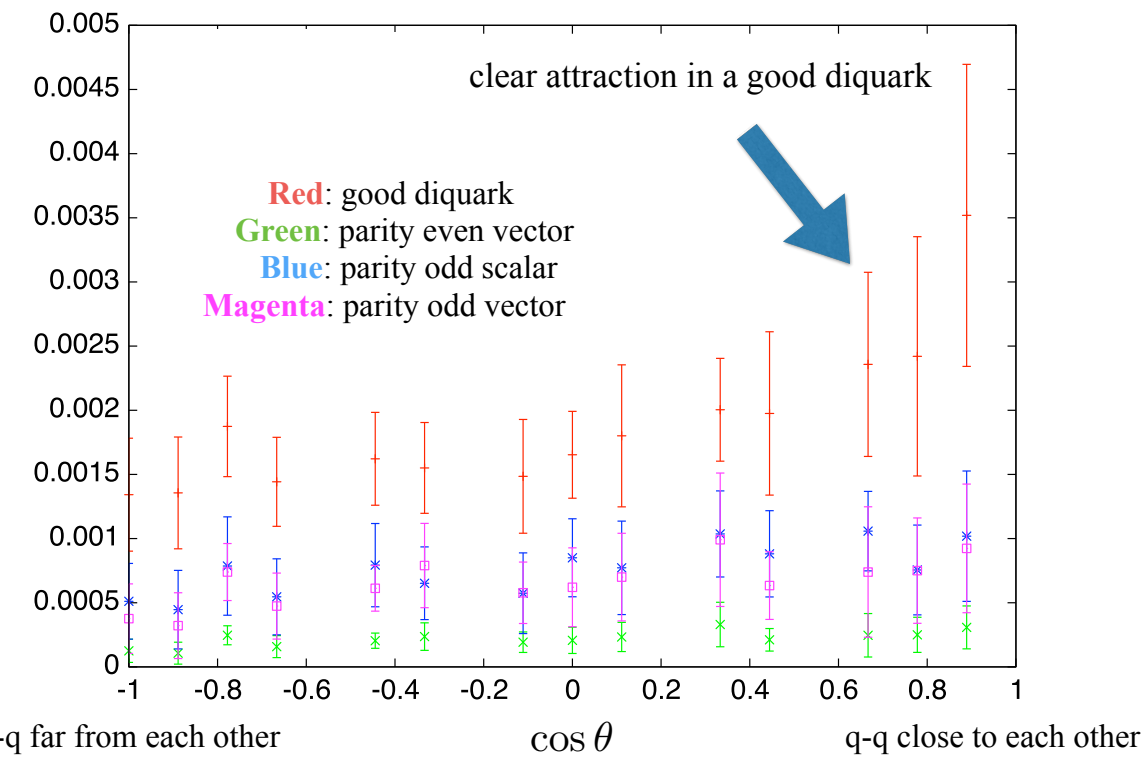

Figure 4: Space distribution of density-density correlator $\left(r=\left|\vec{r}_{1}\right|=\left|\vec{r}_{2}\right|=3 a\right)$

quarks is observed in a good diquark. This statement is consistent with the prediction obtained by phenomenological calculation and the former study based on a lattice QCD simulation for heavier quarks than this study [6]. Therefore we can conclude that lattice QCD simulations support the possibility of existence of diquark states.

\section{Acknowledgments}

We thank the ALPHA collaboration for generously providing CLS gauge configurations. The calculations have been performed on SX-ACE at Osaka University.

\section{References}

[1] M. Anselmino, E. Predazzi, S. Ekelin, S. Fredriksson, and D. B. Lichtenberg, Rev. Mod. Phys. 65 (1993) 1199

[2] LHCb collaboration, Phys. Rev. Lett. 115, 072011 (2015)

[3] R. L. Jaffe, Phys. Rept. 409, 1 (2005)

[4] C. Alexandrou, Ph. de Forcrand, and A. Tsapalis, Phys. Rev. D 66, 094503 (2002)

[5] P. Fritzsch, F. Knechtli, B. Leder, M. Marinkovic, S. Schaefer, R. Sommer, and F. Virotta, Nucl. Phys. B865 (2012) 397

[6] C. Alexandrou, Ph. de Forcrand, and B. Lucini, Phys. Rev. Lett. 97, 222002 (2006) 